\title{
MIMO Terminal Performance Evaluation With a Novel Wireless Cable Method
}

\author{
Wei Fan, Pekka Kyösti, Lassi Hentilä, and Gert Frølund Pedersen
}

\begin{abstract}
Conventional conductive method, where antennas on the device under test (DUT) are disconnected from antenna ports and replaced with radio frequency (RF) coaxial cables, has been dominantly utilized in industry to evaluate multiple-input multiple-output capable terminals. However, direct RF cable connection introduces many practical problems and a radiated method to replace cable connection is highly desirable. Existing wireless cable method relies on the knowledge of a transfer matrix between the channel emulator (CE) output ports and DUT antenna ports, and also requires an anechoic chamber, which might be impractical and expensive. In this paper, a novel wireless cable method is proposed and experimentally validated. By recording the average power (i.e., reference signal received power in the long-term evolution) per DUT antenna port and selecting optimal complex weights at the CE output ports, a wireless cable connection can be achieved. The proposed method can be executed in a small RF shielded anechoic box and offers low system cost, high measurement reliability, and repeatability.
\end{abstract}

Index Terms-Anechoic chambers, antenna measurements, fading channels, radio propagation, testing.

\section{INTRODUCTION}

M ULTIANTENNA technology has been widely utilized, due to its capability to significantly improve wireless system performance in terms of data throughput, quality of service, and cellular coverage, without increasing transmission power and bandwidth [1]. In recent wireless technologies, such as long-term evolution (LTE) and LTE-Advanced, multiple antennas are employed at the terminal side as well [2]. Performance testing is an essential step in different phases of product development, from early stage research prototypes (i.e., proof of concept), design optimization, to actual product approval for the final rollout. Due to complexities with multiantenna terminal designs, a flexible, fast, cost-effective, and accurate testing solution is highly desirable [3]-[6].

A conventional conductive method has been dominantly adopted in industry so far for device performance

This work was supported by the Innovation Fund Denmark via the VIRTUalized envirOnment for Communication System Development and Optimization Project. The work of W. Fan was supported by the Danish Council for Independent Research under Grant DFF611100525. (Corresponding author: Wei Fan.)

W. Fan and G. F. Pedersen are with the Department of Electronic Systems, Faculty of Engineering and Science, Antennas, Propagation and Radio Networking Section, Aalborg University, 9220 Aalborg, Denmark (e-mail: wfa@es.aau.dk).

P. Kyösti and L. Hentilä are with Keysight Technologies Finland Oy, 90590 Oulu, Finland. evaluation [7], [8]. In the conductive testing, antennas on the device under test (DUT) are disconnected from antenna ports and replaced with radio frequency (RF) coaxial cables, i.e., with antennas bypassed. In this way, specified test signals are guided directly to the DUT antenna ports via RF cables in a conductive manner. Conductive testing has been seen as an attractive and acceptable choice in the early stage of chipset and baseband development [9]. However, in a later stage of product Development, it requires breaking or otherwise modifying the DUT and presents many shortcomings in practice [9], [10]. An alternative testing method without cable connection is highly desired.

The over-the-air (OTA) method, which eliminates the need for cable connections, is becoming more important and popular [3]-[8]. Various OTA methodologies have been proposed and discussed in the literature, e.g., the reverberation chamber (RC)-based method [11], [12], the radiated two-stage method [6], [10], [13], [14], and the multiprobe anechoic chamber (MPAC) method [15]. Various methods differ in how spatial propagation channels can be emulated, in which the multiantenna terminals are tested. Furthermore, among the mentioned methods, only the MPAC and radiated twostage methods are shown to be capable of emulating arbitrary spatial channel models even in principle [3], [4], [6]. The wireless industry, through CTIA and 3GPP standardization bodies, has been working on standard multiple-input multipleoutput (MIMO) OTA testing methodologies. MIMO OTA methods aim typically at end-to-end performance testing, i.e., with DUT antennas included. As for the widely adopted conductive testing, true antenna characteristics at the DUT are typically neglected. DUT antenna characteristics, if known, can be embedded in the channel models for performance evaluation as well [10], [16].

With the MPAC method, controllable RF multipath propagation environments can be physically mimicked and controlled at DUT location in a repeatable way, by using a radio channel emulator (CE) connected to an array of probe antennas within an anechoic chamber [15]. One major drawback of the MPAC method is its cost, where a large number of probe antennas (each of which is connected to a CE RF interface channel) and anechoic chambers are required to ensure target channel models can be accurately reproduced over a test area suitable for mobile terminals. For example, to emulate a dual-polarized 2-D standard channel models, e.g., standard spatial channel model-extended (SCME) urban micro (UMi) and SCME urban macro (UMa) [17], eight dual-polarized probe antennas (i.e., $16 \mathrm{CE} \mathrm{RF}$ interface channels) are recommended in the standardization [3], [4]. The dimension of the anechoic 
chamber should also be sufficiently large to ensure that DUT and probe antennas are within each other's far-field zones. Setting up a 3-D MPAC configuration, which is required to emulate more realistic 3-D channel models, necessitates more probe antennas and, therefore, a need for more expensive setups [18]. Furthermore, utilizing MPAC method as a means to evaluate physically large DUTs [e.g., base stations (BSs), cars, and so on] would result in cost-prohibitive designs, due to the large test area requirement.

With the radiated two-stage method, a nonintrusive complex radiation-pattern measurement of receive $(\mathrm{Rx})$ antennas on the DUT is required in the first stage [10], [13], [14]. The measured radiation patterns are then incorporated with target channel models in the $\mathrm{CE}$ in the second stage. To direct signals from each probe antenna to desired DUT antenna port without RF cable connection, a transfer matrix between $\mathrm{CE}$ output ports and DUT antenna ports needs to be measured and calibrated out to achieve a "wireless cable transmission" in the second stage [10], [13], [14], [19]. With the MPAC method, the DUT is evaluated in the emulated RF propagation environments as it would operate in real world. It is therefore a true end-to-end performance evaluation, without a need to know DUT antenna characteristics. However, DUT antenna characteristics need to be completely known in advance and included in channel models for the radiated two-stage method. As a result, it might be problematic with adaptive DUT antenna patterns.

The wireless cable concept is highly attractive. With this concept, the number of required RF interface channels for the $\mathrm{CE}$ is required only to match the number of receive $(\mathrm{Rx})$ antennas on the DUT, regardless of the complexity of the chosen channel model and DUT size [6], [10], [19]. Note that mobile terminals are often equipped with few Rx antennas, due to the small form factor. The wireless cable concept has been discussed for evaluating MIMO handsets in the radiated two-stage method [10] and for electrically large objects [19]. However, the existing method to achieve wireless cable is practically challenging, since there is a need to measure the transfer matrix in an accurate way. For mobile handset testing, an anechoic chamber is required, as detailed in [10]. In this paper, we propose a novel calibration method to achieve wireless cable transmission for MIMO handset testing. The proposed method is attractive, since it does not require a knowledge of the transfer matrix. Furthermore, measurements can be done in any static environment that offers frequency flat transfer matrix over the considered bandwidth (e.g., a small RF shielded anechoic box), which can greatly reduce system cost compared with setups proposed in [10]. Note that the proposed method can be readily applied in the automotive industry to evaluate antenna systems on the cars [20]-[23], though the focus in this paper is on mobile terminals. The main contributions of this paper lie in the following.

1) A novel calibration procedure to achieve a wireless cable transmission is presented.

2) The proposed method is experimentally validated, via comparing measured throughout results in the proposed setup and in the conductive testing.
3) The impact of isolation levels (i.e., a measure of wireless cable connection quality) on measured throughput results is investigated.

4) The impact of branch power radio (BPR) and channel correlation on the measured throughput is investigated based on the proposed wireless cable method.

\section{Method}

For an MIMO system equipped with $M$ transmitter (Tx) and $N$ receiver (Rx) antennas, the time-variant channel frequency response (CFR) is given by $\boldsymbol{H}(f, t) \in \mathbb{C}^{N \times M}$, with $h_{n, m}(f, t)$ the CFR from the $m$ th Tx to the $n$th Rx. The CFR includes both the multipath propagation and the antenna patterns at the Tx and Rx sides.

The MIMO signal model can be written as

$$
\boldsymbol{y}(f, t)=\boldsymbol{H}(f, t) \boldsymbol{x}(f, t)+\boldsymbol{n}(f, t)
$$

where $\boldsymbol{y}(f, t) \in \mathbb{C}^{N \times 1}$ denotes receive signal vector at the $N$ Rx antenna ports, $\boldsymbol{x}(f, t) \in \mathbb{C}^{M \times 1}$ denotes transmit signal vector at the $M$ Tx antenna port, and $\boldsymbol{n}(f, t) \in \mathbb{C}^{N \times 1}$ denotes the noise vector at the $N \mathrm{Rx}$ antenna ports. The objective is to achieve a specified signal vector $\boldsymbol{y}(f, t)$ for relevant MIMO receivers and to evaluate how MIMO receivers operate under the specified signals. Therefore, noise vector is ignored in the study for the sake of simplicity. In this section, we first briefly explain the basic principles of the conductive cable method and the wireless cable method. After that, we propose a new calibration method to achieve the wireless cable transmission. The radiated two-stage method proposed in [10] is named here after as the reference wireless cable method. Note that the main goal of this paper is to replace conventional conductive testing (i.e., cable connection) with a wireless cable method. Therefore, DUT antenna patterns are neglected. As explained earlier, DUT antenna characteristics are typically neglected in conductive testing. However, complex DUT antenna radiation patterns can be included in channel models if available. In the following, we take $2 \times 2 \mathrm{MIMO}$ as an example in the discussion.

\section{A. Problem Statement}

1) Conductive Cable Testing: As shown in Fig. 1, the system consists of a BS emulator, a CE, and a DUT. The BS emulator mimics the cellular network end of the link. The CE performs convolution of Tx signals with target channel impulse responses (CIRs). The RF cables direct the specified signals to the DUT antenna ports, with DUT antennas bypassed. We have

$$
\boldsymbol{y}(f, t)=\boldsymbol{A} \boldsymbol{H}(f, t) \boldsymbol{x}(f, t)
$$

where $\boldsymbol{A} \in \mathbb{C}^{2 \times 2}$ is the transfer matrix, with $a_{i, j}$ the complex transfer coefficient between the $j$ th CE output port and the $i$ th DUT antenna port, $i, j \in[1,2]$. Note that the cable loss and phase delay introduced by the propagation in RF cables can be easily measured and calibrated out in practical measurements. Therefore, for conductive testing, $\boldsymbol{A}=\boldsymbol{I}_{\mathbf{2}}$, with $\boldsymbol{I}_{2} \in \mathbb{R}^{2 \times 2}$ being the identity matrix, can be assumed for ideal cable 


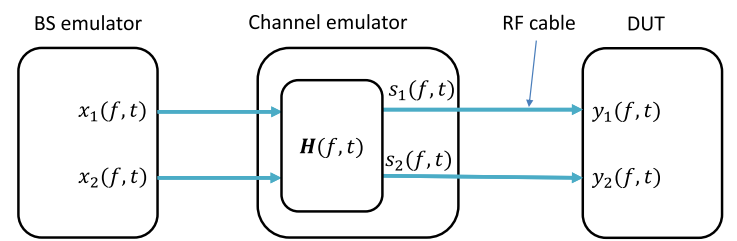

Fig. 1. System diagram of conductive testing for a $2 \times 2$ MIMO system. $\boldsymbol{A}$ denotes the transfer function between the CE output ports and Rx antenna ports. $s_{i}(f, t)$ is the signal intended for the $i$ th $\mathrm{Rx}$ antenna port with $i \in[1,2]$.

connections (i.e., no crosstalk between cables, no cable loss, and no phase delay introduced by the propagation in a cable).

As shown in Fig. 1, the desired situation, where $s_{1}(f, t)$ is received only by $\mathrm{Rx}$ antenna port 1 and $s_{2}(f, t)$ is received only by Rx antenna port 2 , can be easily achieved in the cable testing setup.

Though conceptually simple and widely used in the industry, there are many drawbacks with conductive testing for performance evaluation.

1) Conductive testing requires the opening of the terminal case to get access to antenna connectors. Furthermore, antenna connectors are typically fragile, unstable, and can get broken easily.

2) Antenna connectors might not be available, if antennas are integrated with the RF front-end circuitry, which would prevent the utilization of conducted testing. Furthermore, it is expected that antenna connectors will not be available for future $5 \mathrm{G}$ mobile terminals, where frequency bands are expected to move to millimeterwave bands [24].

3) The test connectors and cables might affect the device electronic performance, e.g., the inconsistency due to impedance mismatch between coaxial cable and connectors [10]. Device self-generated noise and interference cannot be modeled as well [10].

4) Cable measurements are mostly convenient and reliable in conditions where the DUT is equipped with one or few antenna elements only and with the antenna connectors physically available. Now with, e.g., current LTE user equipment (UE), there are typically no antenna connectors available. If connectors have to be soldered for testing purposes to the printed circuit board of the UE, the device gets fragile, and the error probability when sequentially attaching/detaching RF cables increases significantly. Furthermore, for future 5G antenna systems equipped with possibly hundreds of antennas, hundreds of cable connections to the antenna ports and respective hardware would be required, leading to error-prone, complicated, and cost-prohibitive setups.

5) Conductive setups can be cumbersome, especially for $5 \mathrm{G}$ millimeter-wave bands where waveguides are used to replace high lossy RF cables.

6) DUT might be optimized for conducted testing, which will give unrealistic performance results.

2) Wireless Cable Method: A system diagram of the wireless cable method is shown in Fig. 2, where the basic idea is that the transfer matrix $\boldsymbol{A}$ can be calibrated out in the CE to

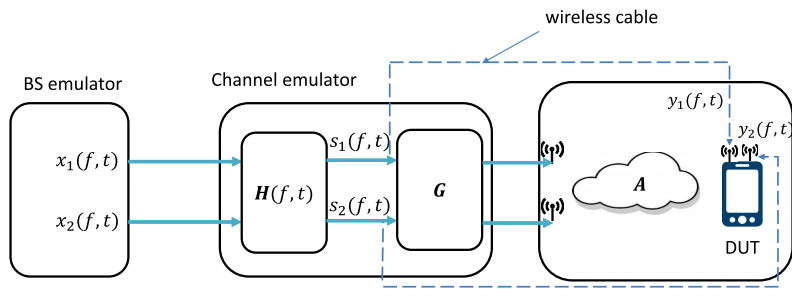

Fig. 2. System diagram of the wireless cable method. Dotted lines: equivalent wireless cable connections.

achieve the wireless cable connection. With the wireless cable method, the received signal vector at DUT antenna port can be expressed as

$$
\boldsymbol{y}(f, t)=\boldsymbol{A} \boldsymbol{G} \boldsymbol{H}(f, t) \boldsymbol{x}(f, t)=\boldsymbol{H}(f, t) \boldsymbol{x}(f, t)
$$

where $\boldsymbol{A}$ is the transfer matrix between the CE output ports and the Rx antenna ports. $\boldsymbol{G}$ is the calibration matrix, which satisfies $\boldsymbol{A} \boldsymbol{G}=\boldsymbol{I}_{N} \in \mathbb{R}^{N \times N}$. $\boldsymbol{G H}(f, t)$ is the channel model implemented in the CE. The objective of wireless cable method is to achieve radiated cable connection. As shown in Fig. 2, for a $2 \times 2$ MIMO case with $K=2$, without compensating the transfer matrix $\boldsymbol{A}$, i.e., with $\boldsymbol{G}=\boldsymbol{I}_{2}$ set, $s_{1}(f, t)$ would be transmitted directly from the OTA antenna 1, causing $s_{1}(f, t)$ to appear at both Rx antenna ports with different amplitude and phase shifts. Similarly, $s_{2}(f, t)$ transmitted from OTA antenna 2 will also be received by both $\mathrm{Rx}$ antenna ports at different amplitudes and phases. With the transfer matrix calibrated out (i.e., $\boldsymbol{A} \boldsymbol{G}=\boldsymbol{I}_{2}$ ), we can ensure that $s_{1}(f, t)$ is received only by Rx antenna port 1 and $s_{2}(f, t)$ is received only by $\mathrm{Rx}$ antenna port 2 , which is equivalent to conducted cable testing, however, without actual RF cable connections. As the method name suggests, we achieve a wireless cable connection.

It is noted that we need to ensure the number of OTA antennas $(K)$ is no less than the number of UE antennas $(N)$ in the system design. To achieve wireless cable connections, we need to ensure that $\boldsymbol{A} \boldsymbol{G}$ approximates $\boldsymbol{I}_{N}$. $\boldsymbol{A} \boldsymbol{G}$ would fail to approximate $\boldsymbol{I}_{N}$ with $K<N$, since it would be rank deficient: $\operatorname{rank}(\boldsymbol{A} \boldsymbol{G}) \leq \min (\operatorname{rank}(\boldsymbol{A}), \operatorname{rank}(\boldsymbol{G})) \leq K<N$, where $\operatorname{rank}()$ denotes the rank operator. For a system with $K \geq N$, if $\boldsymbol{A}$ has full rank, i.e., $\operatorname{rank}(\boldsymbol{A})=N, \operatorname{rank}(\boldsymbol{A} \boldsymbol{G})=N$ can be obtained with $\operatorname{rank}(\boldsymbol{G})=N$.

However, it is not a trivial task to obtain the transfer matrix $\boldsymbol{A}$. In [10], it was proposed that the complex transfer matrix $\boldsymbol{A}$ can be calculated from the complex OTA antenna gains, the complex propagation coefficients from OTA antennas to DUT antennas, and the complex DUT antenna gains. It was mentioned in [10] that it is always possible to have a nonsingular $\boldsymbol{A}$, via rotating the DUT. How accurate the specified signal vector $\boldsymbol{y}(f, t)$ can be reproduced at the DUT antenna ports depends on the measurement accuracy of transfer matrix $\boldsymbol{A}$. However, the reference wireless cable method might be problematic and expensive in practice [10].

1) Accurate knowledge of complex radiation patterns and placement of all OTA antennas is required. The measured complex antenna pattern accuracy might suffer from practical nonidealities, e.g., cable effect. 


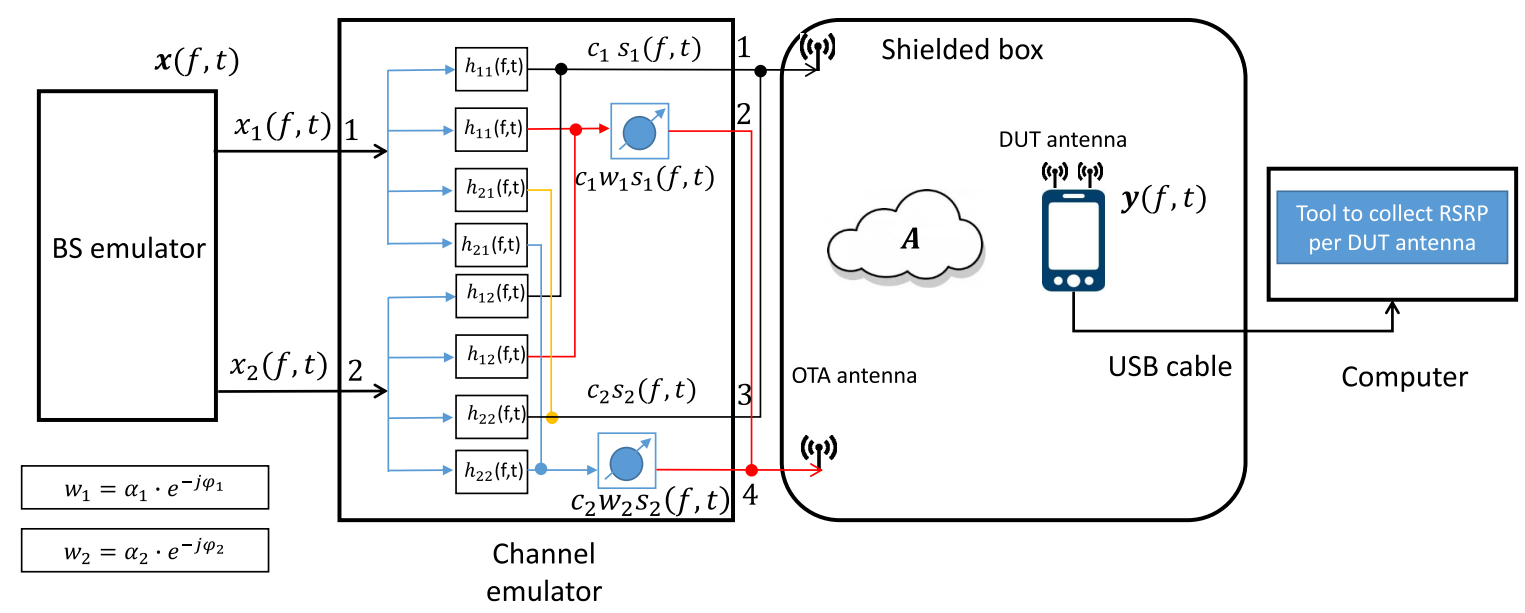

Fig. 3. Illustration of the proposed method for a $2 \times 2$ MIMO system with $K=2$ OTA antennas. The number of CE output ports $L=4$ is set.

2) Accurate knowledge of complex radiation patterns and placement of all DUT antennas is required. A nonintrusive, complex radiation-pattern measurement of DUT antenna patterns is challenging, and it is not currently mandatory in any standard and thus not enabled by all terminal vendors.

3) An ideal line-of-sight propagation (i.e., free space) without any reflection inside the anechoic chamber from OTA antennas to DUT antennas is assumed to calculate the complex propagation coefficients.

With the reference method, an isolation level up to 18 and $20.5 \mathrm{~dB}$ were reported in [13] and [14], respectively. In [19], the wireless cable technique was applied for measuring large objects in a nonanechoic scenario. However, the transfer matrix $\boldsymbol{A}$ was directly measured. This, however, requires access to the DUT antenna connectors, and therefore is not suitable for handset radiated testing.

\section{B. Proposed Wireless Cable Method}

1) System Diagram: The system diagram of the proposed method is shown in Fig. 3. The system consists of a BS emulator with two output ports, a CE with two input ports and four output ports (as numbered in the figure), two external RF combiners (i.e., one combiner connected to $\mathrm{CE}$ output ports 1 and 3 , and another one connected to $\mathrm{CE}$ output ports 2 and 4), a small RF shielded anechoic box, and a DUT. With the proposed method, it is required that the CE should support phase and gain control at the each CE output port. A tool is installed in a computer to collect the average receive power level [i.e., reference signal received power (RSRP) value in LTE] for each DUT antenna. A universal serial bus cable is used to connect the DUT to the computer. Note that the focus of this paper is on the downlink (i.e., from BS to the UE). As for the uplink, one of the OTA antennas connected to the $\mathrm{CE}$ can be utilized to realize uplink connection, where a built-in RF circulator in the CE output port can separate the downlink and uplink signals. The uplink is omitted in Fig. 3 for simplicity.

2) Signal Model: The signal at the CE output ports 1-4 can be expressed as $c_{1} s_{1}(f, t), c_{1} w_{1} s_{1}(f, t), c_{2} s_{2}(f, t)$, and $c_{2} w_{2} s_{2}(f, t)$, respectively, with

$$
\left[\begin{array}{l}
s_{1}(f, t) \\
s_{2}(f, t)
\end{array}\right]=\boldsymbol{H}(f, t) \boldsymbol{x}(f, t)
$$

where $w_{1}$ is the complex weight allocated to one of the two ports transmitting $s_{1}(f, t)$ (i.e., 1 or 2$)$ and $w_{2}$ is the complex weight allocated to one of the two ports transmitting $s_{2}(f, t)$ (i.e., 3 or 4), as shown in Fig. 3. $c_{1}$ and $c_{2}$ denote the gain weight to set the power level for the first and second wireless cable connection, respectively, as discussed later.

The received signal vector at DUT antenna ports can be expressed as

$$
\boldsymbol{y}(f, t)=\boldsymbol{A} \underbrace{\left[\begin{array}{cc}
c_{1}\left(\begin{array}{c}
1 \\
w_{1}
\end{array}\right) & \left.c_{2}\left(\begin{array}{c}
1 \\
w_{2}
\end{array}\right)\right]
\end{array}\right] \boldsymbol{H}(f, t)} \boldsymbol{x}(f, t) .
$$

Note that matrix in the curly bracket is the channel model implemented in the CE. $\boldsymbol{A}\left[c_{1}\left(\begin{array}{c}1 \\ w_{1}\end{array}\right) c_{2}\left(\begin{array}{c}1 \\ w_{2}\end{array}\right)\right] \boldsymbol{H}(f)$ is a time-variant matrix containing weights of signals at $\mathrm{CE}$ output ports, the time-variant radio channel model $\boldsymbol{H}(f, t)$, and the static transfer matrix $\boldsymbol{A}$ inside the RF shielded anechoic box (including effects of OTA antennas, multipath propagation inside the RF shielded anechoic box, DUT antennas, OTA antennas, cables, and connectors).

For notational clarity, we name

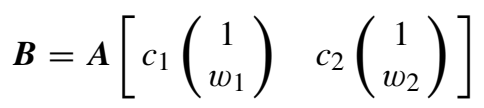

with its $(i, j)$ th entry as $b_{i, j}$. To achieve wireless cable transmission, we need to ensure in all cases the specified signal vector $\boldsymbol{y}(f, t)=\boldsymbol{H}(f, t) \boldsymbol{x}(f, t)$ at the DUT antenna ports. This can be achieved if matrix $\boldsymbol{B}$ can be forced into an identity matrix, via selecting complex weights $w_{1}$ and $w_{2}$, and gain weights $c_{1}$ and $c_{2}$. The objective function can be written as

$$
\min _{c_{1}, c_{2}, w_{1}, w_{2}}\left\|\boldsymbol{A}\left[c_{1}\left(\begin{array}{c}
1 \\
w_{1}
\end{array}\right) c_{2}\left(\begin{array}{c}
1 \\
w_{2}
\end{array}\right)\right]-\left[\begin{array}{ll}
1 & 0 \\
0 & 1
\end{array}\right]\right\| .
$$

As shown in Fig. 4, which is an equivalent system diagram of the proposed method shown in Fig. 3, we have the following. 


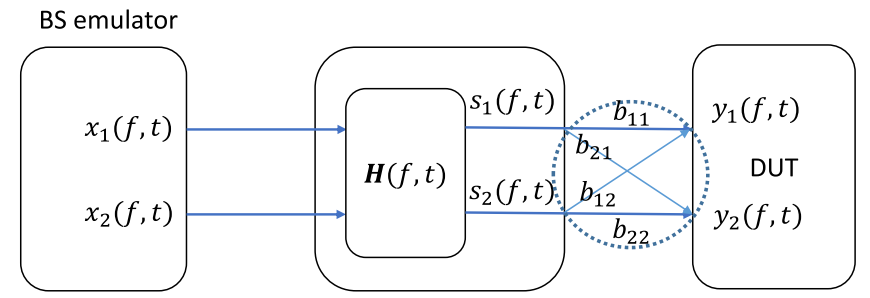

Fig. 4. Equivalent system diagram of the proposed method shown in Fig. 3.

1) $b_{11}$ denotes the propagation coefficient for the desired direct link from $s_{1}(f, t)$ to $y_{1}(f, t)$, and $b_{22}$ for the desired direct link from $s_{2}(f, t)$ to $y_{2}(f, t)$.

2) $b_{21}$ denotes the propagation coefficient for the undesired crosstalk from $s_{1}(f, t)$ to $y_{2}(f, t)$, and $b_{12}$ for the undesired crosstalk from $s_{2}(f, t)$ to $y_{1}(f, t)$.

With a known calibration matrix $\boldsymbol{A}$, complex weights $w_{1}$ and $w_{2}$ and gain weight $c_{1}$ and $c_{2}$ can be directly solved in (7), similar to the reference wireless cable method. As discussed in Section II-A2, it is difficult to obtain calibration matrix $\boldsymbol{A}$ accurately in practice.

3) Calibration Procedure: In this section, we describe a novel calibration procedure to determine complex weights $w_{1}$ and $w_{2}$, and gain weights $c_{1}$ and $c_{2}$. Wireless cable connections can be achieved under two conditions.

1) Crosstalks $b_{21}$ and $b_{12}$ are minimized.

2) $b_{11}$ and $b_{22}$ are balanced to ensure a BPR $0 \mathrm{~dB}$ between the two DUT antenna ports.

Inspired by this, a novel calibration method is proposed to achieve the wireless cable transmission, as detailed in the following.

1) Locate the DUT in an RF shielded anechoic box.

2) Set channel models in a bypassed mode in the CE (i.e., $\left.\boldsymbol{H}=\boldsymbol{I}_{2}\right)$, and establish a call in the spatial multiplexing mode.

3) Find optimal complex weight $w_{1}$ to establish the first wireless cable transmission from $s_{1}$ to $y_{1}$.

a) Enable CE output ports transmitting $s_{1}$ and $w_{1} s_{1}$ (i.e., ports 1 and 2), and disable CE output ports transmitting $s_{2}$ and $w_{2} s_{2}$ (i.e., ports 3 and 4). Gain weight $c_{1}$ is set to a value that offers good signal quality at the Rx antenna ports.

b) The RSRP values at the two Rx antenna ports with only $s_{1}$ active are

$$
\begin{aligned}
& R S R P_{1}\left(w_{1}\right)=\left|c_{1}\right|^{2}\left|b_{11}\right|^{2} P\left(x_{1}\right) \\
& \operatorname{RSRP} P_{2}\left(w_{1}\right)=\left|c_{1}\right|^{2}\left|b_{21}\right|^{2} P\left(x_{1}\right)
\end{aligned}
$$

where $P\left(x_{1}\right)$ is the average power level for the Tx signal $x_{1}$. According to (6), we have $b_{11}=$ $c_{1}\left(a_{11}+w_{1} a_{12}\right)$ and $b_{21}=c_{1}\left(a_{21}+w_{1} a_{22}\right)$. Note that RSRP values at the two $\mathrm{Rx}$ antenna ports can be recorded with available tools installed in a computer. The optimal complex weight $w_{1}$ can be obtained in two steps.

i) Set $w_{1}=\alpha_{o} \exp \left(-j \varphi_{1}\right)$ (i.e., gain term fixed to $\alpha_{o}$ ), with $\varphi_{1}$ sweeping within $\left[0^{\circ}, 360^{\circ}\right]$. Record $\operatorname{RSR} P_{2}\left(w_{1}\right)$ and store phase $\hat{\varphi}_{1}$ value

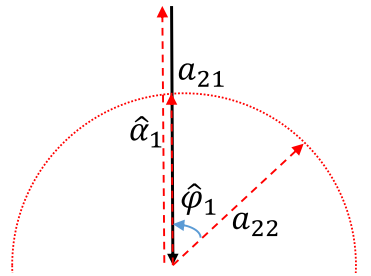

Fig. 5. Illustration of the two-step search procedure.

that gives minimal $R S R P_{2}\left(w_{1}\right)$. As shown in Fig. 5, an optimal $\hat{\varphi}_{1}$ is found when $a_{22}$ and $a_{21}$ have a phase difference of $180^{\circ}$.

ii) Set $w_{1}=\alpha_{1} \exp \left(-j \hat{\varphi}_{1}\right)$ (i.e., phase term fixed to $\left.\hat{\varphi}_{1}\right)$, with $\alpha_{1}$ sweeping within a suitable range around $\alpha_{o}$. Record $R S R P_{2}\left(w_{1}\right)$ and store the gain term $\hat{\alpha}_{1}$ value that gives minimal $R S R P_{2}\left(w_{1}\right)$. As shown in Fig. 5, an optimal $\hat{\alpha}_{1}$ value is found when $a_{11}$ and $a_{12}$ have a phase difference of $180^{\circ}$ and equal amplitude. Note that a proper $\alpha_{o}$ is selected to ensure that all swept $\alpha_{1}$ values in the range can be supported by the $\mathrm{CE}$ with a good signal dynamic range.

As we can see, a complicated 2-D exhaustive search (i.e., both amplitude and phase term) can be simplified to two 1-D monotonic searches.

4) Similar to step 3, we can find the optimal complex weight $w_{2}=\hat{\alpha}_{2} \exp \left(-j \hat{\varphi}_{2}\right)$ to establish the second wireless cable transmission from $s_{2}$ to $y_{2}$. Similarly, we need to enable CE output ports transmitting $s_{2}$ and $w_{2} s_{2}$ (i.e., ports 3 and 4), and disable CE output ports transmitting $s_{1}$ and $w_{1} s_{1}$ (i.e., ports 1 and 2). It is noted that gain weight $c_{2}$ should be set to a value that offers good signal quality at the Rx antenna ports. Furthermore, we need to record RSRP values at the two Rx antenna ports with only $s_{2}$ active, i.e., $R S R P_{1}\left(w_{2}\right)$ and $R S R P_{2}\left(w_{2}\right)$. $w_{2}$ is found when minimal $\operatorname{RSR} P_{1}\left(w_{2}\right)$ is achieved. The isolation levels for the two wireless cable transmissions can be defined as

$$
\begin{aligned}
& I_{1}=\frac{R S R P_{1}\left(w_{1}\right)}{R S R P_{2}\left(w_{1}\right)} \\
& I_{2}=\frac{R S R P_{2}\left(w_{2}\right)}{R S R P_{1}\left(w_{2}\right)} .
\end{aligned}
$$

5) Analogous to ensure the same cable loss for cables in the conductive testing, we need to ensure that propagation coefficients for the desired direct links (i.e., $b_{11}$ and $b_{22}$ ) are balanced. Balanced branches can be achieved via adjusting gain weight $c_{i}$ for $i \in[1,2]$ as

$$
\operatorname{RSRP} P_{1}\left(w_{1}\right)=\operatorname{RSRP} P_{2}\left(w_{2}\right) .
$$

In this step, a BPR $=0 \mathrm{~dB}$ is targeted. As shown later, different BPR values for MIMO performance testing can be realized, via selecting attenuation coefficients for the wireless cable. Note that balancing branch power would not affect the isolation between the two wireless cable connections, since complex weights $w_{1}$ and $w_{2}$ are not altered. 


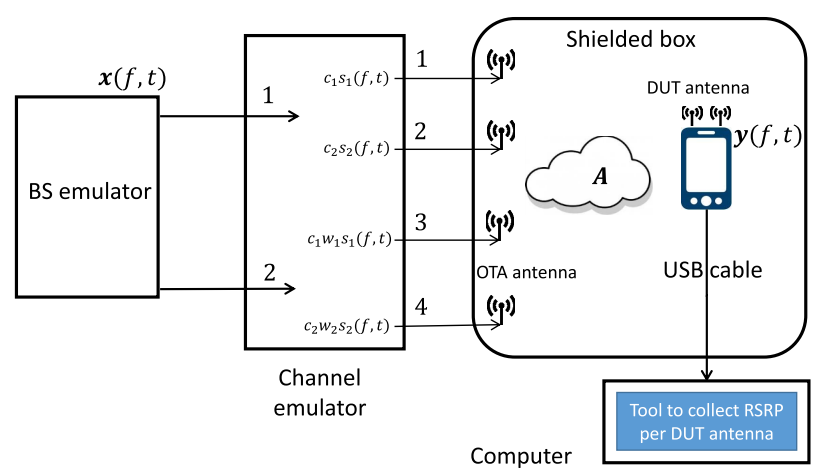

Fig. 6. System diagram of a $2 \times 2$ MIMO system with the number of OTA antennas $K=4$. Compared with setup proposed in Fig. 3, there is no need for external RF combiners. Furthermore, it offers another dimension of optimization (i.e., across routing matrices).

6) After the calibration stage, a wireless cable connection (i.e., low crosstalk and balanced branches) is established, via setting the complex weight $w_{1}$ and $w_{2}$ and gain weight $c_{1}$ and $c_{2}$ for each $\mathrm{CE}$ output port. We can perform actual throughout measurements with desired channel models $\boldsymbol{H}(t, f)$. Similar to the reference method in [10], the DUT stayed untouched during the whole test, which implies that a fully automated testing can be utilized. The calibration procedure and the actual performance testing with specified channel models can be performed smoothly, without operator intervention.

\section{Setup With $K>N$}

We have discussed the proposed method for setup with $K=N=2$. In this section, we discuss cases, where we have more OTA antennas than DUT antennas, i.e., $K>N$.

As mentioned earlier, it is likely that a good wireless cable transmission cannot be established in some unfavorable conditions. With $K=N$, a favorable condition can be found via altering the transfer matrix $\boldsymbol{A}(f)$ following a trial and error process, e.g., relocating or rotating the DUT, repositioning OTA antennas, or e.g., putting intentional metallic reflectors inside the RF shielded anechoic box. However, this would necessitate changes in the setup. With $K>N$, we have the flexibility to search for the optimal routing matrix that presents the best wireless cable connection. Routing matrix defines how signals $s_{1}(f, t)$ and $s_{2}(f, t)$ are linked to OTA antennas in the CE. For $2 \times 2 \mathrm{MIMO}$ with $K=2$, as shown in Fig. 3, there is no flexibility for routing, since the number of signals is the same as the number of OTA antennas. In this section, we take $2 \times 2$ MIMO with $K=4$ as an example in the discussion, as shown in Fig. 6.

The received signal vector at DUT antenna ports can be expressed as

$$
\boldsymbol{y}(f, t)=\boldsymbol{A} \boldsymbol{R} \boldsymbol{s}(f, t)
$$

where $\boldsymbol{A} \in \mathbb{C}^{2 \times 4}$ is the transfer matrix from $\mathrm{CE}$ output ports to DUT antenna ports and $\boldsymbol{R} \in \mathbb{C}^{4 \times 2}$ is the routing matrix within the $\mathrm{CE}$.
For $2 \times 2$ MIMO with $K=4$ as shown in Fig. 6, we have two channels and four OTA antennas. Therefore, we have the flexibility to assign signals $s_{1}(f, t)$ and $s_{2}(f, t)$ to the four CE output ports. The routing matrix $\boldsymbol{R}$ defines on how signals $s_{1}(f, t)$ and $s_{2}(f, t)$ are assigned to CE output ports. With $K=4$, the four CE output ports are divided to two groups, each with two ports. One group transmitting $s_{1}(f, t)$ and $w_{1} s_{1}(f, t)$ and the other two ports transmitting $s_{2}(f, t)$ and $w_{2} s_{2}(f, t)$ as a group. Therefore, we have three possibilities for the routing matrix $\boldsymbol{R}$, that is

$$
\boldsymbol{R}=\left\{\left[\begin{array}{cc}
1 & 0 \\
w_{1} & 0 \\
0 & 1 \\
0 & w_{2}
\end{array}\right],\left[\begin{array}{cc}
1 & 0 \\
0 & 1 \\
w_{1} & 0 \\
0 & w_{2}
\end{array}\right],\left[\begin{array}{cc}
1 & 0 \\
0 & 1 \\
0 & w_{2} \\
w_{1} & 0
\end{array}\right]\right\}
$$

where the second routing matrix $\boldsymbol{R}$ is shown in Fig. 6, i.e., ports 1 and 3 for $s_{1}(f, t)$ and $w_{1} s_{1}(f, t)$ as a group, and ports 2 and 4 for $s_{2}(f, t)$ and $w_{2} s_{2}(f, t)$ as another group, respectively. Similar to the discussions in Section II-B2, a wireless cable transmission can be achieved if $\boldsymbol{A}(f) \boldsymbol{R}(f)$ approximates the identity matrix $\boldsymbol{I}_{2}$. We can then follow the calibration procedure as discussed in Section II-B3 to find optimal complex weights and attenuation weights for each routing matrix. The routing matrix that offers best wireless cable transmission quality (i.e., with the lowest crosstalks) can be selected. As we can see, it is beneficial to have a CE that supports flexible routing (i.e., channel models can be flexible swapped in the $\mathrm{CE}$ ).

With $K>N$, we can select the routing matrix that presents the best wireless cable connection, thus effective reducing the probability of unfavorable conditions. Furthermore, the setups remain the same during the whole measurement. The more OTA antennas we have, the better chances we can avoid unfavorable transfer matrix $\boldsymbol{A}$, with the cost of longer time needed for the calibration stage. In this paper, we have discussed a $2 \times 2$ MIMO system with $K=2$ or 4 . The principle, however, can be easily extended to a general $N \times M$ MIMO system with $K$ OTA antennas $(K \geqslant N)$.

\section{Discussion}

In the above discussion, a frequency flat transfer matrix $\boldsymbol{A}$ over the considered LTE bandwidth is assumed. The proposed wireless cable method only works when the transfer matrix $\boldsymbol{A}$ is frequency flat over the considered LTE bandwidth. As detailed in Section II-B, the basic procedure of the proposed method is that we sweep the complex weight $w_{1}$ and $w_{2}$ allocated to the CE output port and monitor the received RSRP value per DUT port. Since we only have the measured RSRP value available for each DUT port for the full LTE band, we can only obtain one value for each weight $w_{1}$ and $w_{2}$ over the whole band. If the transfer matrix $\boldsymbol{A}$ is frequency selective in the band, we need a different $w_{1}$ and $w_{2}$ values to achieve the wireless cable connection for each frequency sample inside the LTE band. This is not feasible, since one RSRP value per frequency sample is not available in the LTE throughput measurement. This narrowband assumption might be violated when a DUT has a large system bandwidth or 
the utilized RF shielded enclosure is highly reflective (e.g., a metallic RC). This narrowband requirement is easy to meet in practical measurements. In this paper, the considered LTE system bandwidth is rather narrow, i.e., up to $20 \mathrm{MHz}$. Furthermore, either an anechoic chamber or a small anechoic shielded anechoic box equipped with absorbers that provide high attenuation is utilized in this paper, as detailed in the measurement Section III-A.

There are cases where transfer function $\boldsymbol{A}$ is not favorable. As explained in Section II-B, to achieve a wireless cable connection, we need to ensure that $\boldsymbol{A}\left[c_{1}\left(\begin{array}{c}1 \\ w_{1}\end{array}\right) c_{2}\left(\begin{array}{c}1 \\ w_{2}\end{array}\right)\right]$ approximates an identity matrix. In the calibration process, this can be approximated when we have good isolation level and balanced power branch between the wireless cable connections. To have a good isolation level, we need to ensure $\left(\left|c_{1}\left(a_{11}+w_{1} a_{12}\right)\right|^{2} /\left|c_{1}\left(a_{21}+w_{1} a_{22}\right)\right|^{2}\right) \rightarrow \infty$ and $\left(\left|c_{2}\left(a_{21}+w_{2} a_{22}\right)\right|^{2} /\left|c_{2}\left(a_{11}+w_{2} a_{12}\right)\right|^{2}\right) \rightarrow \infty$. This is not achievable if transfer function $\boldsymbol{A}$ is rank deficient (i.e., $a_{11}=$ $a_{21}$ and $a_{12}=a_{22}$ ). Furthermore, we might need to sweep a large range to obtain the optimal amplitude term in the complex weight $w_{1}$ and $w_{2}$, which might be not supported due to the limited dynamic range in the CE output port. Furthermore, it takes long calibration time to sweep a large amplitude range. As discussed, we can find a favorable condition via altering the transfer function $\boldsymbol{A}$. Furthermore, as discussed in Section I, UE antenna patterns are not inherently included in the cable testing and wireless cable. We can embed the UE antenna patterns in the channel models in the performance testing once they are completely known in advance and static. However, this is a problem if UE antennas are adaptive, i.e., UE antennas can adapt to the RF propagation environments. Therefore, it is not possible to evaluate UEs with adaptive antennas with the proposed wireless cable method.

\section{Measurement EQUiPMEnT AND SETUP}

\section{A. Measurement System}

The measurement system is shown in Fig. 6 and detailed in Table I. Note that the DUT internal antennas were replaced by an external LTE mock-up antenna to support multiband measurements [26], though results in one band are shown in this paper. Notice that the testing can be performed in any static environment that offers frequency flat transfer matrix $\boldsymbol{A}$ in principle. An RF shielded enclosure is preferred, since it is cost-effective and can exclude external unwanted interference and noises. In this paper, to validate the robustness of the proposed calibration procedure, calibration measurements in two different scenarios were performed, one in an RF shielded anechoic box and the other in an anechoic chamber, as detailed in Table I. The calibration results for the anechoic chamber and the RF shielded anechoic box were detailed in Sections III-B and IV-A, respectively. Note that throughout measurements were performed only in the RF shielded anechoic box. A photograph of the measured mobile station in an RF shielded anechoic box is shown in Fig. 7. To check whether the transfer matrix $\boldsymbol{A}$ is frequency flat within the LTE bandwidth inside the RF shielded anechoic enclosure, a coherence bandwidth was measured.
TABLE I

SETUP AND SPECIFICATIONS OF EACH COMPONENT IN THE MEASUREMENT SySTEM

\begin{tabular}{|c|c|}
\hline Component & Setup and specifications \\
\hline $\begin{array}{l}\text { Radio } \\
\text { channel } \\
\text { emulator }\end{array}$ & $\begin{array}{l}\text { - Model: Keysight Propsim F32 } \\
\text { - Channel models: The Kronecker channel models } \\
\text { with different channel correlation coefficients } \\
(\rho=\{0,0.6,0.7,0.8,0.9,1\}) \text { were utilized [27], } \\
\text { [28]. Note that same correlations are set at the Tx } \\
\left.\text { and Rx side (i.e. } \rho_{T x}=\rho_{R x}\right) \text {. } \\
\text { - Different BPR levels were realized via attenuating } \\
\text { the CE output powers associated with one wireless } \\
\text { cable. }\end{array}$ \\
\hline $\begin{array}{c}\text { BS } \\
\text { emulator }\end{array}$ & $\begin{array}{l}\text { - Model: Anritsu MT8820C } \\
\text { - Modulation and coding (MCS) index: } 13 \\
\text { - Frame structure: frequency division duplex (FDD) } \\
\text { - LTE frequency band: } 3 \\
\text { - Channel bandwidth: } 10 \mathrm{MHz} \\
\text { - Transmission mode: } 2 \times 2 \text { open loop MIMO }\end{array}$ \\
\hline $\begin{array}{l}\text { Tool to } \\
\text { collect } \\
\text { RSRP per } \\
\text { antenna } \\
\end{array}$ & $\begin{array}{l}\text { - The Keysight Nemo Outdoor tool is installed in a } \\
\text { computer to collect RSRP values per DUT antenna } \\
\text { during the measurement. }\end{array}$ \\
\hline $\begin{array}{l}\text { Measurement } \\
\text { scenario }\end{array}$ & $\begin{array}{l}\text { - Anechoic chamber: A 3D probe configuration with } \\
16 \text { dual-polarized OTA antennas (i.e. four on the } \\
\text { elevation ring with } 30^{\circ} \text { elevation angle, eight on the } \\
\text { azimuth plane, and four on the elevation ring with } \\
-30^{\circ} \text { elevation angle) was present in the anechoic } \\
\text { chamber. The vertically polarized ports of the four } \\
\text { antennas on the elevation ring with } 30^{\circ} \text { elevation } \\
\text { angle were connected to the CE in the study as OTA } \\
\text { antennas. } \\
\text { RF shielded anechoic box: Ramsey STE3600 RF } \\
\text { test shielded enclosure, with interior dimension } \\
28 \mathrm{~cm} \times 40.64 \mathrm{~cm} \times 58.4 \mathrm{~cm}\end{array}$ \\
\hline $\begin{array}{c}\text { OTA } \\
\text { antenna }\end{array}$ & $\begin{array}{l}\text { - Anechoic chamber: ETS-lindgren dual-polarized } \\
\text { wideband Vivaldi antenna. } \\
\text { - RF shielded anechoic box: TESCOM TC-93060A } \\
\text { left hand circularly polarized antenna. }\end{array}$ \\
\hline DUT & - Model: Samsung Galaxy S4. \\
\hline
\end{tabular}

We connect one port of a vector network analyzer (VNA) to one of the probe antennas and the other port of the VNA to a wideband antenna located in the center of the RF shielded anechoic enclosure. We recorded the complex transmission coefficients $S_{21}(f)$ at the center frequency of $1.8425 \mathrm{GHz}$ with a span of $500 \mathrm{MHz}$ for each of the four probe antennas. For each transmission coefficient, a total of 1601 frequency points were stored. An inverse Fourier transform was then performed to obtain the CIRs between the probe antenna and the antenna placed in the center of the RF shielded anechoic enclosure. The measured CIRs are shown in Fig. 8. From the measured CIRs, we calculated the delay spread $\delta_{\tau}$ with a dynamic range of $40 \mathrm{~dB}$. As we can see, the delay spread $\delta_{\tau}$ ranges from 2.3 to $3.2 \mathrm{~ns}$, and the calculated coherence bandwidth $C_{\mathrm{BW}}=1 / \delta_{\tau}$ is more than $300 \mathrm{MHz}$. The coherence bandwidth in the RF shielded anechoic enclosure is much larger than the LTE system bandwidth. Therefore, we can assume a frequency flat transfer matrix $\boldsymbol{A}$ within the LTE bandwidth.

\section{B. Measured Results for the Calibration Stage}

In this section, an example of measured results for the calibration stage in the anechoic chamber is demonstrated. 


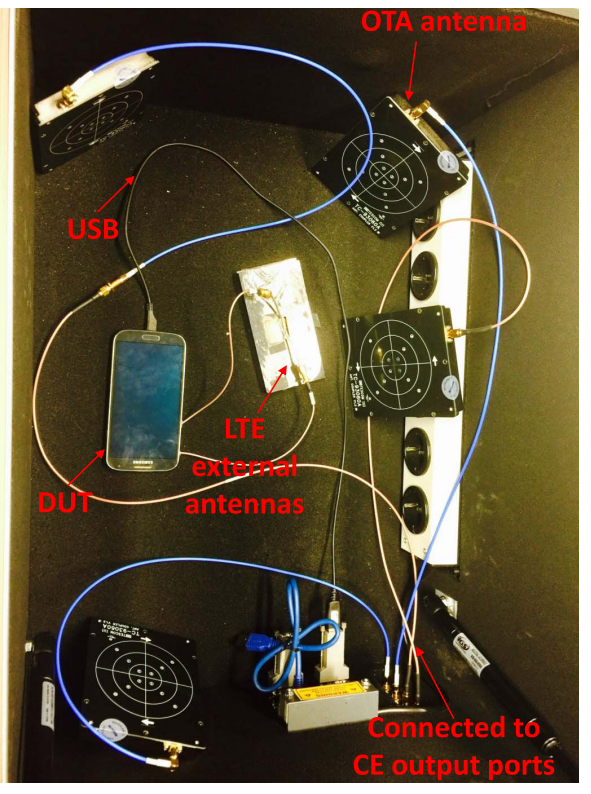

Fig. 7. Photograph of the measurement setup in the RF shielded anechoic box.

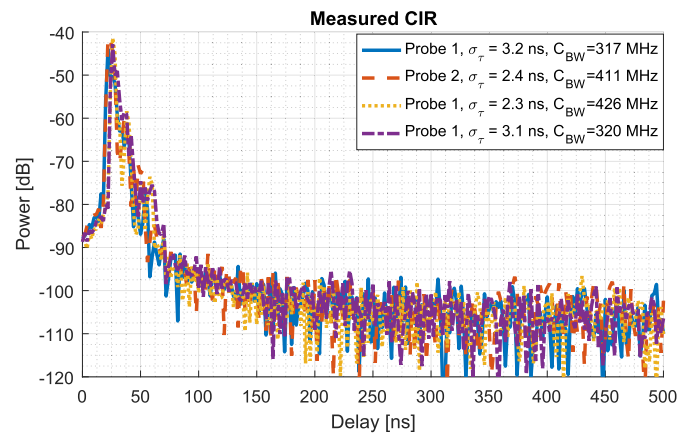

Fig. 8. Measured CIRs in the RF shielded anechoic enclosure.

In the phase tuning stage, we set $w_{1}=\alpha_{o} \exp \left(-j \varphi_{1}\right)$ and $w_{2}=\alpha_{o} \exp \left(-j \varphi_{2}\right)$, with $\alpha_{o}=-6 \mathrm{~dB} . \varphi_{1}$ and $\varphi_{2}$ were swept within $\left[-200^{\circ}, 200^{\circ}\right]$ with a $2^{\circ}$ step. The RSRP values measured in the phase tuning stage for each routing matrix are shown in Fig. 9. Routing matrix $\boldsymbol{R}_{1}$ offers the best isolation performance, with an isolation level of $11.1 \mathrm{~dB}$ achieved for the worse wireless cable connection $\left(I_{1}=19.8 \mathrm{~dB}\right.$ and $I_{2}=$ $11.1 \mathrm{~dB}$ ). The determined optimal phase tuning values are $\hat{\varphi}_{1}=96^{\circ}$ and $\hat{\varphi}_{2}=88^{\circ}$, respectively. Note that for routing matrix $\boldsymbol{R}_{2}$, a negative isolation (i.e., crosstalk stronger than the direct link) was present for all phase tuning values.

After the phase tuning stage, the best routing matrix $\boldsymbol{R}_{1}$ was selected. In the gain tuning stage, we set $w_{1}=\alpha_{1} \exp \left(-j \hat{\varphi}_{1}\right)$ and $w_{2}=\alpha_{1} \exp \left(-j \hat{\varphi}_{1}\right)$, with $a_{1}$ and $a_{2}$ swept within $[-11,0] \mathrm{dB}$ with a $1-\mathrm{dB}$ step. The determined optimal gain tuning values are $\hat{a}_{1}=-5 \mathrm{~dB}$ and $\hat{\varphi}_{2}=-7 \mathrm{~dB}$, respectively, as shown in Fig. 10. An isolation level of $17.6 \mathrm{~dB}$ achieved for the worse wireless cable connection. Note that an isolation level of $13.5 \mathrm{~dB}$ for the worse wireless cable before gain tuning was shown in Fig. 10, which is different from the achieved $11.1 \mathrm{~dB}$ reported in Fig. 9. This is mainly due to the measurement uncertainties for the cross-links. The measured RSRP values for cross-links at optimal phase tuning values
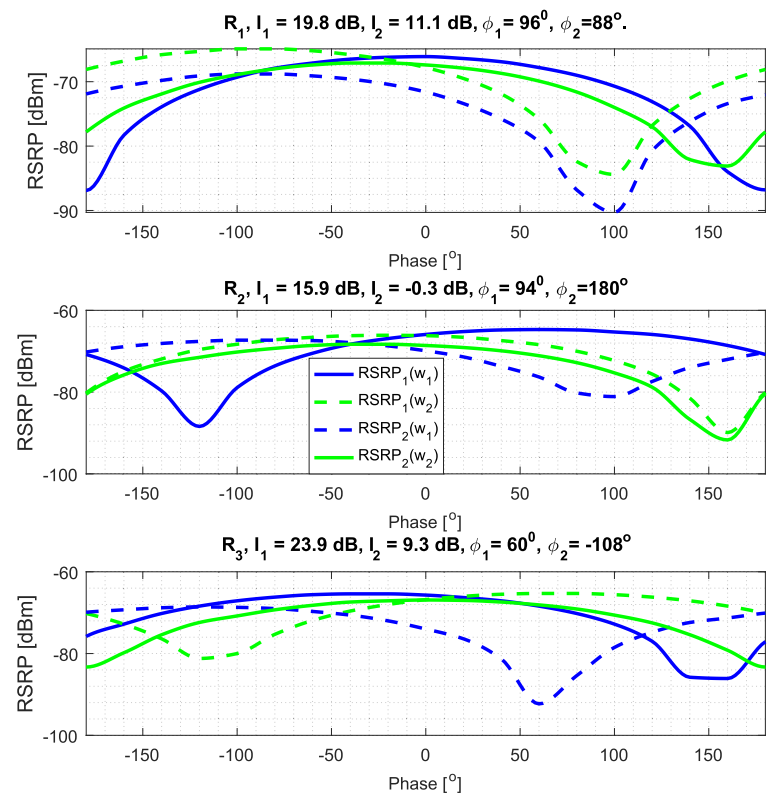

Fig. 9. Measured RSRP value per DUT antenna port in the phase tuning stage for first (top), second (middle), and third (bottom) routing matrices.

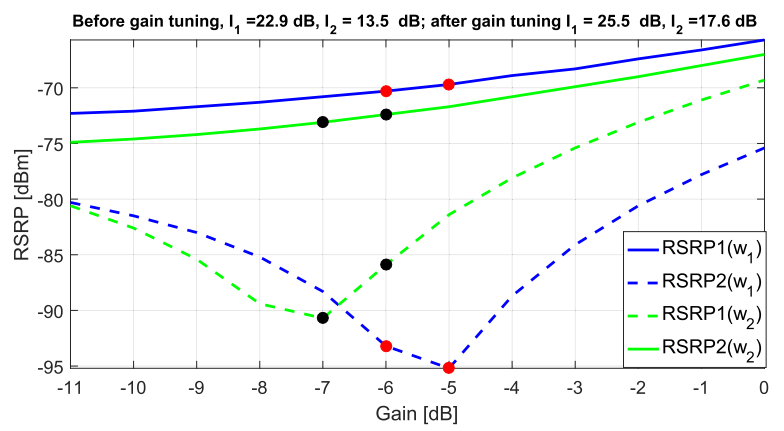

Fig. 10. Measured RSRP value per DUT antenna port in the gain tuning stage.

are small, which are susceptible to system nonidealities, e.g., noise and interference. This is, however, not the case for the direct link at optimal phase tuning value due to its high signal strength. After the gain tuning stage, the isolation levels can be further improved, as expected. As seen in Fig. 10, the branch power is not balanced, with a higher RSRP value for the first wireless cable connection (i.e., the red dots). To balance the two branches, the first wireless connection should be attenuated, as discussed earlier.

Finally, the measured RSRP value per DUT antenna port after the branch balancing stage is shown in Table II. The isolation levels achieved for the two wireless cable connections are $I_{1}=25.5 \mathrm{~dB}$ and $I_{2}=17.6 \mathrm{~dB}$, respectively. Furthermore, the two branches are balanced, with $P\left(y_{1}\right)=-73.1 \mathrm{dBm}$ and $P\left(y_{2}\right)=-73.0 \mathrm{dBm}$, respectively.

Note that it took around $3 \mathrm{~min}$ to complete the whole calibration procedure in the measurements.

\section{Throughput Measurement Procedure}

After the calibration stage, a wireless cable transmission is achieved. The wireless cable connection will be maintained during the throughput measurement, as long as complex 
TABLE II

SETup AND SPECIFICATIONS OF EACH COMPONENT IN THE MEASUREMENT SYSTEM

\begin{tabular}{|c|c|c|c|}
\hline$R S R P_{1}\left(w_{1}\right)$ & $R S R P_{2}\left(w_{1}\right)$ & $R S R P_{1}\left(w_{2}\right)$ & $R S R P_{2}\left(w_{2}\right)$ \\
\hline$-73.1 \mathrm{dBm}$ & $-98.6 \mathrm{dBm}$ & $-90.7 \mathrm{dBm}$ & $-73.1 \mathrm{dBm}$ \\
\hline
\end{tabular}

weights for all $\mathrm{CE}$ output ports are unaltered. The throughput measurement procedure is detailed in [3]. Note that the interference and noise are not modeled in the CE for simplicity. We can perform the MIMO throughput performance testing under arbitrary channel and noise conditions, once a wireless cable connection is established. In the conductive case, the additive white Gaussian noise is generated independently for each CE output. Knowing the power level of CE input signal convolved with the sequence of CIRs, it is possible to adjust the generated noise power to achieve desired signal-to-noise ratio (SNR). Finally, desired $\boldsymbol{H}(f, t) \boldsymbol{x}(f, t)+\boldsymbol{n}(f, t)$ value can be fed to the DUT by RF cable. This works the same way for the proposed wireless cable setup. As we know, MIMO terminal performance is highly sensitive to noise conditions in real channels. Measured throughput generally decreases as the SNR drops [25]. In the MPAC setup, noises radiated by the OTA antennas can be generated as uncorrelated in the CE. However, noises received at different UE antennas might be spatially correlated. Note that this is not a problem for the proposed wireless cable method, since uncorrelated noise can be directly guided to each UE antenna port.

During the throughput measurement, we first load specified channel models in the CE. We then attenuate all CE output ports with a common coefficient $(\gamma \mathrm{dB})$ to achieve RSRP values required for maximum throughput. Note that a common scaling coefficient for all CE output ports will not affect the quality of the wireless cable connection. The RSRP value shown in throughput curves is an average of the RSRP values at two DUT antennas [3]. For each throughput measurement point, 20000 subframes per stream were utilized, as suggested in [3]. After the first throughput measurement point was done, RSRP values were decreased with a $2-\mathrm{dB}$ step, via attenuating all $\mathrm{CE}$ output ports with a common $(\gamma+2) \mathrm{dB}$ coefficient, and then, we repeat the throughput measurement. We continue to reduce the RSRP values with a 2-dB step and repeat the throughput measurement until the LTE throughput reaches below $10 \%$ of the maximum throughput. Note that we use throughput percent, defined as the throughput normalized by its maximum value (i.e., $20.6 \mathrm{Mb} / \mathrm{s}$ ) in this paper, for the sake of easy exhibition. The measurement time depends directly on measurement settings, e.g., the number of measured throughput points, number of subframes per stream, and so on. With the specified settings in the measurement campaign, it took around $3 \mathrm{~min}$ to obtain each throughput curve. As mentioned earlier, throughput measurement results reported in this paper were all performed in the RF shielded anechoic box, as shown in Fig. 7.

\section{Measured Results}

\section{A. Calibration Results for the RF Shielded Anechoic Box}

The achieved isolation level of the best routing matrix with phase tuning in the calibration stage in the RF shielded

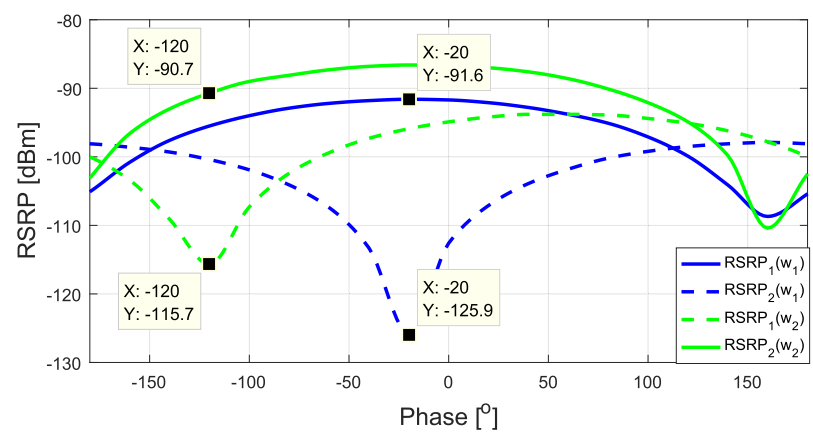

Fig. 11. Measured RSRP value per DUT antenna port during phase sweep The determined optimal phase is $\hat{\varphi}_{1}=-20^{\circ}$ and $\hat{\varphi}_{2}=-118^{\circ}$, respectively.

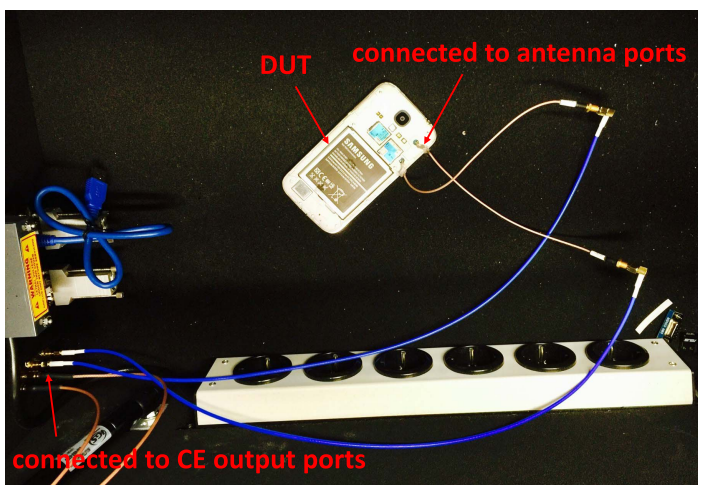

Fig. 12. Photograph of the conductive setup in the RF shielded anechoic box.

anechoic box is shown in Fig. 11. The achieved isolation is $I_{1}=34.3 \mathrm{~dB}$ and $I_{2}=25 \mathrm{~dB}$, respectively. Note that the gain tuning stage was skipped, since a good isolation level had achieved already with only phase tuning.

\section{B. Validation Measurement}

To experimentally validate the proposed wireless cable technique, measured throughput results in the proposed setup were compared with results achieved in the conductive setup. The measurement system for the conductive setup is shown in Fig. 1, and a photograph is shown in Fig. 12. Similar to the throughput measurement procedure for the wireless cable setups, we decreased the RSRP values at the DUT antennas in the conductive setup for each throughput measurement point, via attenuating two $\mathrm{CE}$ output ports connected to the two DUT antennas with a common attenuation coefficient in the conductive testing. Note that different attenuation coefficients would introduce branch power difference, which would affect the measured MIMO throughput performance.

Two representative $2 \times 2$ MIMO channel models, i.e., one uncorrelated with $\rho=0$ and the other fully correlated with $\rho=1$, are selected in the validation measurements. As explained earlier, BPR $=0 \mathrm{~dB}$ was set both for the conductive and the wireless cable setup in the validation measurements. The validation measurement results are shown in Fig. 13. The measured throughput in uncorrelated channels is higher than in fully correlated channels with the same RSRP values, as expected. With the uncorrelated channel model, maximum throughput can be achieved with an average RSRP 


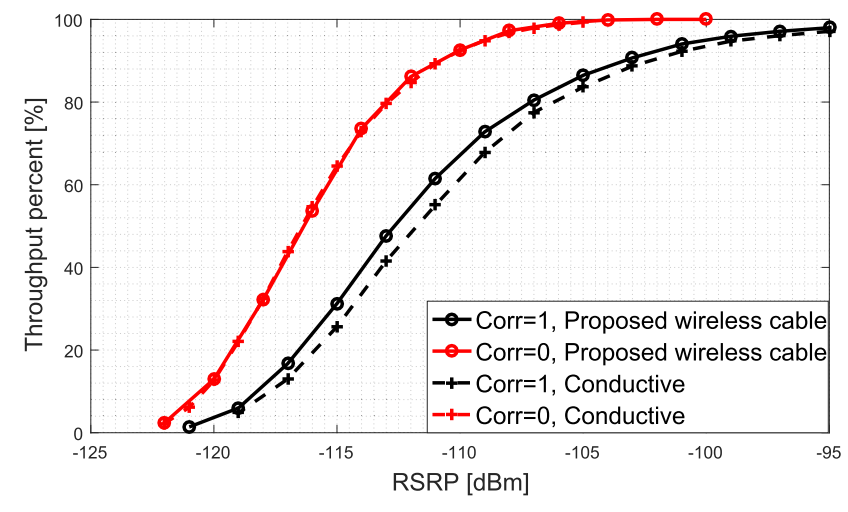

Fig. 13. Measured throughput results in uncorrelated channels and fully correlated channels in the conductive and proposed setups.

value around $-106 \mathrm{dBm}$. However, maximum throughput is much more difficult to achieve in the fully correlated channel, where an average RSRP value of more than $-95 \mathrm{dBm}$ is needed.

For the uncorrelated channel model, an excellent agreement is achieved for the throughput results measured in the conductive and the proposed setup, as shown in Fig. 13. A small deviation between the measured throughput results exists in the two setups in fully correlated channels. The difference in RSRP values is up to $0.5 \mathrm{~dB}$ at $70 \%$ maximum throughput. The channel correlation properties might be slightly altered due to measurement uncertainty in the system. MIMO throughput is highly sensitive to channel correlation in the high correlation region (e.g., $\rho>0.7$ ) and not sensitive to channel correlation values in the low correlation region $(\rho<0.5)$, as shown later. With $\rho=1$, the channel correlation can be easily decorrelated due to system nonidealities, which would affect measured throughput results. Throughput measurement results in the uncorrelated channel model are more tolerable to system nonidealities. The validation measurement results demonstrated that the proposed setup can replace the conductive setup.

\section{Impact of Isolation Levels}

To utilize the proposed method in practice, it is of importance to know the impact of a degraded isolation level on the measured throughput results. The goal is to determine the level of isolation needed to ensure a good wireless cable transmission quality. A similar study was reported for the reference method in [14], where various isolation levels were achieved via modifying the inverse transfer matrix $\boldsymbol{A}^{+}$with errors. Results demonstrated that with an isolation level around $14 \mathrm{~dB}$, an RSRP error of around $0.5 \mathrm{~dB}$ at $70 \%$ maximum throughput can be observed. The considered channel models are the SCME UMa and the SCME UMi channel models. However, it is not clear whether branch powers are still balanced after introducing errors in $\boldsymbol{A}^{+}$. In this paper, two wireless cables were balanced to achieve BPR $=0 \mathrm{~dB}$, as explained in the calibration stage. Therefore, the throughput difference is only introduced by different channel correlations in channel models. The crosstalk between wireless cables would essentially change the channel correlations.

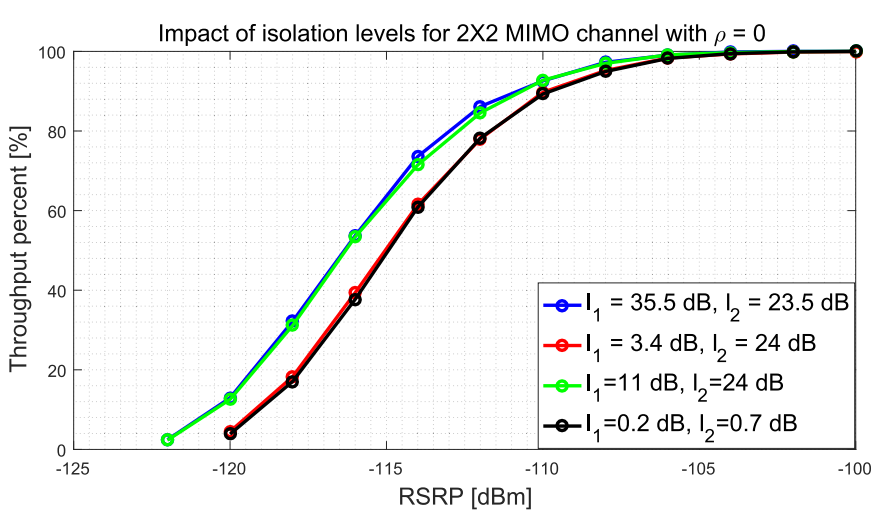

Fig. 14. Impact of isolation levels for the uncorrelated MIMO channel model.

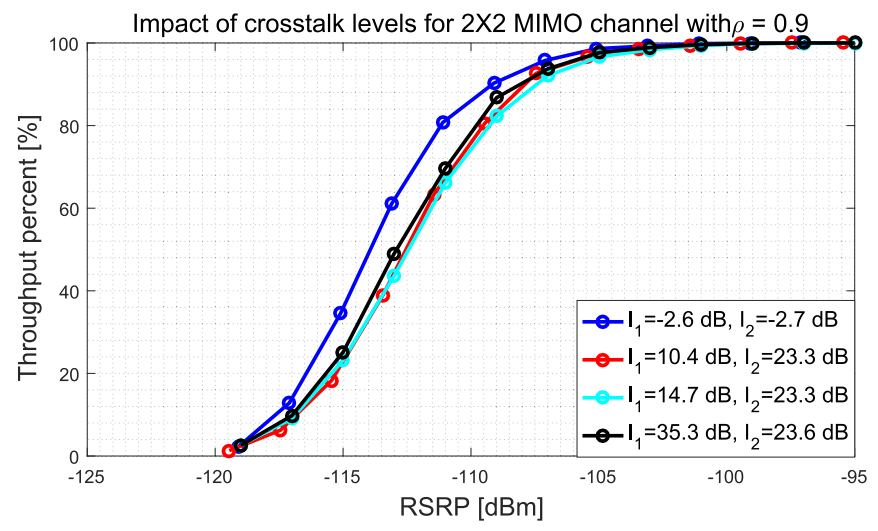

Fig. 15. Impact of isolation levels for the high MIMO channel model.

The impact of isolation levels for the uncorrelated MIMO channel model (i.e., $\rho=0$ ) and a high correlated channel model (i.e., $\rho=0.9$ ) is shown in Figs. 14 and 15 , respectively. Due to lack of measurement time, only four isolation conditions were investigated for each channel model. An isolation level around $I_{1}=35.5 \mathrm{~dB}$ and $I_{2}=23.5 \mathrm{~dB}$ was best achieved for the two wireless cable transmissions with optimal complex weights $w_{1}$ and $w_{2}$, respectively. The other degraded isolation values can be achieved via selecting different complex weights, as shown in Section III-B.

For the uncorrelated channel model in Fig. 14, we set $w_{2}=\alpha_{0} \exp \left(-j \hat{\varphi}_{2}\right)$ fixed and select three different $\varphi_{1}$ values to achieve three isolation levels for the first wireless cable, i.e., 3.4, 11, and $35.5 \mathrm{~dB}$, respectively. Note that the small difference in $I_{2}$ in different throughput measurements is due to the fact that the RSRP value of the cross-link was low and, therefore, sensitive to noise and interference, as explained earlier. With degraded isolation levels, the measured throughput results tend to get worse, since crosstalks would correlate the uncorrelated channel. It can be seen that a degraded isolation, with a level of $11 \mathrm{~dB}$, has negligible impact on throughput. A degraded isolation case with low isolation levels $\left(I_{1}=0.2 \mathrm{~dB}\right.$ and $\left.I_{22}=0.7 \mathrm{~dB}\right)$ for both wirelesscable connections is shown as well (black curve in Fig. 14). An error in the RSRP value of around $1.5 \mathrm{~dB}$ at $70 \%$ maximum throughput is observed. 


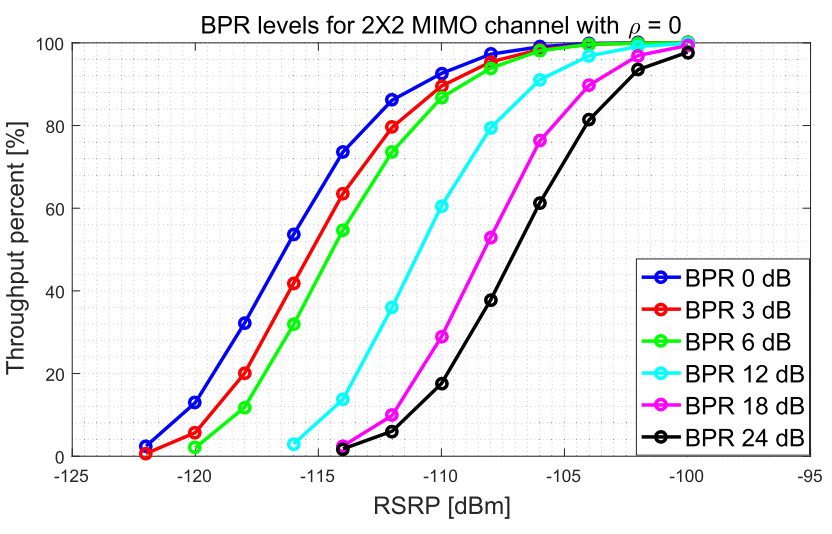

Fig. 16. Impact of different BPR levels on measured throughput.

For the high correlated channel model, a similar analysis was performed. We set $w_{2}$ with optimal phase value and select three different $\varphi_{1}$ values to achieve three isolation levels for the first wireless cable, i.e., 10.4, 14.7, and $35.3 \mathrm{~dB}$, respectively. Unlike the uncorrelated case, the measured throughput result tends to get better with degraded isolation levels. This is due to the fact that crosstalks would decorrelate the high correlated channel. The measured throughput result is slightly affected with an isolation level of $I_{1}=10.4 \mathrm{~dB}$, with a difference in RSRP values less than $0.5 \mathrm{~dB}$ at $70 \%$ maximum throughput.

Based on the measurement results, we can conclude that an isolation level of around $11 \mathrm{~dB}$ should be sufficient in practical setup to establish a good wireless cable.

\section{Performance Analysis of a Commercial MIMO Handset}

Low channel correlation and low BPR between antenna branches are two factors that are commonly aimed for when designing MIMO terminals. In this section, the impact of channel correlation coefficients and BPRs on measured throughput is investigated based on the proposed wireless cable technique.

1) $B P R$ : As explained earlier, an isolation level of $I_{1}=$ $34.3 \mathrm{~dB}$ and $I_{2}=25 \mathrm{~dB}$ with $\mathrm{BPR}=0 \mathrm{~dB}$ can be achieved for the two wireless cables with the calibration stage. Different BPR levels can be achieved, via attenuating one of the two wireless cables at the $\mathrm{CE}$ output ports. For example, to obtain a $\mathrm{BPR}=3 \mathrm{~dB}$, we can set $3-\mathrm{dB}$ attenuation to the first wireless cable (i.e., two CE ports transmitting $s_{1}$ and $w_{1} \cdot s_{1}$ attenuated with $3 \mathrm{~dB}$ ).

The impact of different BPR levels on measured throughput results for an uncorrelated channel model is shown in Fig. 16. The measured throughput performance deteriorates, as the BPR level increases, as expected. Compared with the curve with $\mathrm{BRP}=0 \mathrm{~dB}$, a difference in RSRP values around 1 and $10 \mathrm{~dB}$ is observed at $70 \%$ maximum throughput for $\mathrm{BPR}=3$ and $24 \mathrm{~dB}$, respectively.

2) Channel Correlation: The impact of different channel correlations on measured throughput results with $\mathrm{BPR}=0 \mathrm{~dB}$ is shown in Fig. 17. The measured throughput performance gets worse with higher channel correlation coefficient, as expected. Compared to the curve with $\rho=0$, a difference in RSRP values less than 1 and around $5 \mathrm{~dB}$ is observed at $70 \%$ maximum throughput for $\rho=0.6$ and $\rho=1$, respectively. Furthermore, the higher the channel correlation, the larger

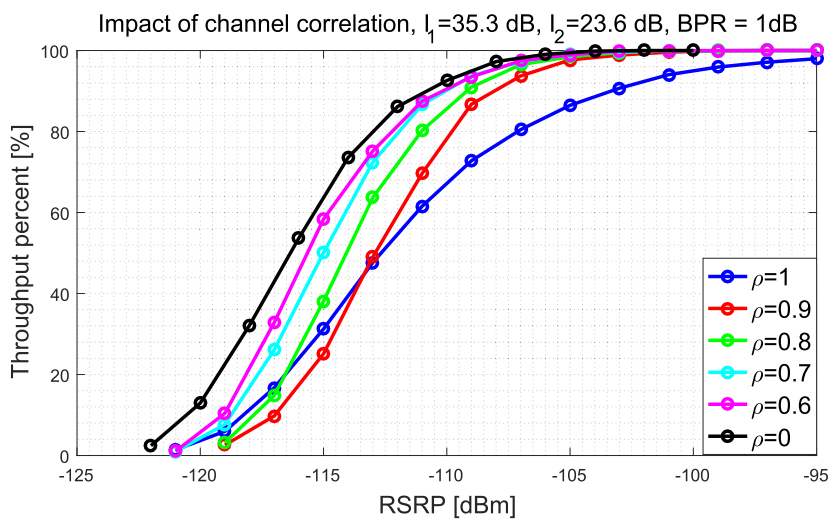

Fig. 17. Impact of different channel correlations on measured throughput.

the RSRP value required to achieve maximum throughput. The impact of channel correlation and BPR on the shape of throughput curve is different.

\section{CONCLUSION}

A novel wireless cable method is proposed in this paper for MIMO capable terminal performance testing. The proposed method utilized the recorded RSRP value per DUT antenna to determine the optimal complex weights needed to achieve a wireless cable, thereby avoiding the need to measure the transfer matrix between the CE ports and DUT antenna ports. Furthermore, the method can be executed in any static environment, without a need for anechoic chambers. The proposed method is experimentally validated, via comparing measured throughput results in the proposed setup and the conductive setup. A deviation in the RSRP value up to $0.5 \mathrm{~dB}$ at $70 \%$ maximum throughput can be achieved between the two setups. An isolation level up to $25 \mathrm{~dB}$ between the wireless cable connection can be achieved in an RF shielded anechoic box with only phase tuning in the calibration stage. The impact of a degraded isolation level on the measured throughput results is investigated for the uncorrelated $(\rho=0)$ and high correlated $(\rho=0.9)$ MIMO channel, respectively. The measured throughput results showed that an isolation level of around $11 \mathrm{~dB}$ should be sufficient in practical setup to establish a good wireless cable. The impact of channel correlation and BPR values on measured throughput is investigated based on the proposed method. BPR measured results showed that a difference in RSRP values around 1 and $10 \mathrm{~dB}$ is observed at $70 \%$ maximum throughput for $\mathrm{BPR}=3$ and $24 \mathrm{~dB}$, compared to the throughput curve with $\mathrm{BRP}=0 \mathrm{~dB}$, respectively. As for the channel correlation analysis, a difference in RSRP values around $5 \mathrm{~dB}$ is observed at $70 \%$ maximum throughput for $\rho=1$, compared to the throughput curve with $\rho=0$.

\section{REFERENCES}

[1] M. A. Jensen and J. W. Wallace, "A review of antennas and propagation for MIMO wireless communications," IEEE Trans. Antennas Propag., vol. 52, no. 11, pp. 2810-2824, Nov. 2004.

[2] C. Hoymann et al., "LTE release 14 outlook," IEEE Commun. Mag., vol. 54, no. 6, pp. 44-49, Jun. 2016.

[3] "Test plan for $2 \times 2$ downlink MIMO and transmit diversity over-the-air performance," version 1.1, CTIA Certification, Tech. Rep., Aug. 2016.

[4] Verification of Radiated Multi-Antenna Reception Performance of User Equipment (UE), document TR 37.977 V14.0.0, 3rd Generation Partnership Project, Jun. 2016. 
[5] M. Rumney, R. Pirkl, M. H. Landmann, and D. A. Sanchez-Hernandez, "MIMO over-the-air research, development, and testing," Int. J. Antennas Propag., vol. 2012, May 2012, Art. no. 467695, doi: 10.1155/2012/ 467695.

[6] MIMO Over the Air (OTA) Handset Performance and Testing, Santa Rosa, CA, USA, Keysight Technol., Jul. 2015.

[7] Testing 5G: Evolution or Revolution, Santa Rosa, CA, USA, Keysight Technol., Oct. 2016.

[8] Modelling What Matters: Keeping a Correct Focus on 5G, Santa Rosa, CA, USA, Keysight Technol., Oct. 2016.

[9] Over-The-Air Testing of Receive Diversity and MIMO Capable Terminals, Microw. Vis. Group (MVG), Paris, France, Jul. 2015.

[10] W. Yu, Y. Qi, K. Liu, Y. Xu, and J. Fan, "Radiated two-stage method for LTE MIMO user equipment performance evaluation," IEEE Trans. Electromagn. Compat., vol. 56, no. 6, pp. 1691-1696, Dec. 2014.

[11] X. Chen, "Throughput modeling and measurement in an isotropicscattering reverberation chamber," IEEE Trans. Antennas Propag., vol. 62, no. 4, pp. 2130-2139, Apr. 2014.

[12] X. Chen, "Experimental investigation and modeling of the throughput of a $2 \times 2$ closed-loop MIMO system in a reverberation chamber," IEEE Trans. Antennas Propag., vol. 62, no. 9, pp. 4832-4835, Sep. 2014.

[13] M. Rumney, H. Kong, Y. Jing, and X. Zhao, "Advances in antenna pattern-based MIMO OTA test methods," in Proc. 9th Eur. Conf. Antennas Propag. (EuCAP), May 2015, pp. 1-5.

[14] M. Rumney, H. Kong, Y. Jing, Z. Zhang, and P. Shen, "Recent advances in the radiated two-stage MIMO OTA test method and its value for antenna design optimization," in Proc. 10th Eur. Conf. Antennas Propag. (EuCAP), Apr. 2016, pp. 1-5.

[15] P. Kyösti, T. Jämsä, and J.-P. Nuutinen, "Channel modelling for multiprobe over-the-air MIMO testing," Int. J. Antennas Propag., vol. 2012, Mar. 2012, Art. no. 615954, doi: 10.1155/2012/615954.

[16] I. Szini, G. Pedersen, A. Tatomirescu, and A. Ioffe, "MIMO $2 \times 2$ absolute data throughput concept," in Proc. 7th Eur. Conf. Antennas Propag. (EuCAP), Mar. 2013, pp. 299-302.

[17] Spatial Channel Model for Multiple Input Multiple Output (MIMO) Simulations, document TR 25.996 V13.0.0, 3rd Generation Partnership Project, Dec. 2015.

[18] C. Schirmer et al., "3D wave-field synthesis for testing of radio devices," in Proc. 8th Eur. Conf. Antennas Propag. (EuCAP), Apr. 2014, pp. 3394-3398.

[19] C. Schirmer, M. Lorenz, W. A. T. Kotterman, R. Perthold, M. H. Landmann, and G. D. Galdo, "MIMO over-the-air testing for electrically large objects in non-anechoic environments," in Proc. 10th Eur. Conf. Antennas Propag. (EuCAP), Apr. 2016, pp. 1-6.

[20] M. G. Nilsson, P. Hallbjörner, N. Arabäck, B. Bergqvist, T. Abbas, and F. Tufvesson, "Measurement uncertainty, channel simulation, and disturbance characterization of an over-the-air multiprobe setup for cars at $5.9 \mathrm{GHz}$," IEEE Trans. Ind. Electron., vol. 62, no. 12, pp. 7859-7869, Dec. 2015.

[21] M. Nilsson, P. Hallbjörner, N. Arabäck, B. Bergqvist, and F. Tufvesson, "Multipath propagation simulator for V2X communication tests on cars," in Proc. 7th Eur. Conf. Antennas Propag. (EuCAP), Apr. 2013, pp. $1342-1346$.

[22] R. He, A. F. Molisch, F. Tufvesson, Z. Zhong, B. Ai, and T. Zhang, "Vehicle-to-vehicle propagation models with large vehicle obstructions," IEEE Trans. Intell. Transp. Syst., vol. 15, no. 5, pp. 2237-2248, Oct. 2014.

[23] R. He et al., "Characterization of quasi-stationarity regions for vehicleto-vehicle radio channels," IEEE Trans. Antennas Propag., vol. 63, no. 5, pp. 2237-2251, May 2015.

[24] G. M. Rebeiz, "Millimeter-wave and terahertz integrated circuit antennas," Proc. IEEE, vol. 80, no. 11, pp. 1748-1770, Nov. 1992.

[25] Inter-Lab/Inter-Technique OTA Performance Comparison Testing for MIMO Devices, document MOSG120521R4, CTIA Certification, Aug. 2012.

[26] S. S. Zhekov, A. Tatomirescu, and G. F. Pedersen, "Compact multiband sensing MIMO antenna array for cognitive radio system," in Proc. Loughborough Antennas Propag. Conf. (LAPC), Nov. 2015, pp. 1-5.

[27] J.-P. Kermoal, L. Schumacher, K. I. Pedersen, P. E. Mogensen, and F. Frederiksen, "A stochastic MIMO radio channel model with experimental validation," IEEE J. Sel. Areas Commun., vol. 20, no. 6, pp. 1211-1226, Aug. 2002.

[28] L. Schumacher and B. Dijkstra, "Description of a Matlab implementation of the indoor MIMO WLAN channel model proposed by the IEEE 802.11 TGn channel model special committee," Implement. Note Version, vol. 5, pp. 1-27, May 2004. 